\title{
Thin film mechanical characterization of UV-curing acrylate systems
}

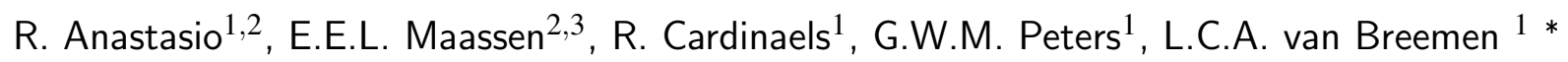

${ }^{1}$ Department of Mechanical Engineering, Materials Technology Institute, Eindhoven University of Technology, P.O.Box 513, 5600 MB, Eindhoven, the Netherlands

2 Brightlands Materials Center (BMC), 6167 RD Geleen, the Netherlands

${ }^{3}$ Department of Chemical Engineering and Chemistry, Eindhoven University of Technology,P.O.Box 513, $5600 \mathrm{MB}$, Eindhoven, the Netherlands

${ }^{*}$ Author to whom correspondence should be addressed. E-mail: L.C.A.v.Breemen@tue.nl Tel: $+31(0) 402473092$ 


\begin{abstract}
This study presents the mechanical characterization of UV-curing acrylate systems. UV-curable polymers are commonly used in the stereolithography (SLA) technique to build multi-layered objects. Typically, the mechanical properties of the 3D-printed product are affected by the intrinsic material heterogeneity along the sample thickness. To understand what determines this heterogeneity, single layers of UV-curable polymer are characterized and the effect of process conditions on the mechanical properties is studied. Micro-compression experiments are carried out to determine the intrinsic mechanical properties which are representative of one single UV-cured layer. To determine the right conditions to generate maximally-cured micropillars, the evolution with irradiation time of monomer conversion, glass-transition temperature and yield stress has first been studied. Thereto, micrometer-sized pillars and dog-bone shaped samples have been prepared via UV-curing. Micro-compression measurements on maximally-cured micropillars are performed to study possible size effects. The results reveal that with decreasing pillar size, the yield stress decreases. Tensile measurements are performed on dog-bone shaped samples which have been processed in the same way as compared to the compression samples. These tensile tests show higher yield stress values when compared with compression tests. This size effect can be attributed to the rinsing with acetone during the sample preparation that leads to a removal of monomer from the crosslinked network. As a consequence, in the real 3D-printing process, the mechanical properties will depend on the feature size. In conclusion, a method is presented to determine the mechanical properties of one single layer of material used in the rapid-prototyping SLA process. The experimental procedure we adopted requires only a few millilitres of material and, therefore, is well suited for screening materials under real SLA process conditions.
\end{abstract}




\section{Introduction}

In recent years, several rapid prototyping technologies, commonly known as 3D printing, have been developed to revolutionize the way in which products are designed, fabricated, and processed. These techniques are intended to improve the competitiveness, flexibility, and accuracy of final products and to reduce the lead time of small components and, mainly, of complex objects [1]. Stereolithography (SLA) is an additive manufacturing process in which the products are built from a photocurable liquid starting material. The 3D-printer includes a resin bath which contains the photopolymer, a UV-laser beam and a support platform that can be moved in the height direction. The UV-laser supplies energy to induce the photo-polymerization process, and consequently the irradiated material is converted into the solid state. The resin is composed of a mixture of monomers and photo-initiators. The desired pattern is exposed to the UV-light that is absorbed by the initiator, free radicals are formed and the polymerization of the liquid material takes place [2]. After the first layer is created, the platform moves by the cured-layer thickness and new layers of resin are added. The final three-dimensional objects are removed from the platform, cleaned with a solvent and post-curing treatments are carried out to enhance the final properties. Due to the presence of internal stresses and different curing levels in each layer, post-curing treatments lead to a disproportionate shrinkage of the 3D-printed object [3]. The printing process, although apparently simple, is rather complicated and challenging to understand: the mechanical and thermal properties of the final product are strictly correlated to the polymerization kinetics of each layer, homogeneity of the monomer conversion, layer adhesion and environmental conditions. For instance, common UV-curable resins are known for their high sensitivity to oxygen inhibition. Decker et al. [4] have carried out experiments on acrylate and epoxy systems, and revealed that in the presence of air, the conversion is progressively slowing down because of the inhibitory effect of the atmospheric oxygen on the radical polymerization of acrylate monomers.

The most straightforward way to study the mechanical properties, life-time expectations and deformation phenomena in polymers, is to perform tensile or compression tests under different loading conditions [5, 6], for which millimeter-sized specimens are usually required. In the SLA process, products are created layer-by-layer and each layer is of micrometer dimensions. Hence, to study the mechanical properties of a UV-curable polymer, a multi-layered sample has to be prepared to achieve the desired final thickness and, due to the SLA process, the final result can be affected by an unequal curing along the sample thickness [7]. Therefore, studying properties of one single layer is the first step to understand the entire process. In particular, to study the intrinsic response of the UV-curable resin, uniaxial compression tests are required, in which the material is deformed homogeneously up to a large plastic deformation ( $70 \%$ strain). Compression is a viable alternative for determination of linear and non-linear mechanical properties, that entails several advantages over tensile testing. It provides the intrinsic mechanical response as necking and delocalization are prevented. In addition, compression tests can easily be performed on small and thin samples. The nano-indentation technique is generally employed to probe mechanical properties of small amounts of material and it is largely used for elastic modulus and hardness determination. Moreover, it is used to perform compression tests on micromachined pillars made of metallic materials, see for example [8]. Wang et al. [9] proposed the use of the nano-indenter to perform micro-compression experiments on focused ion beam (FIB) milled epoxy micropillars. In the same way, Guruprasad et al. [10] have conducted micro-compression tests on glassy PS micropillars fabricated by hot embossing. This process by which the pillars have been prepared, 
leads to different cooling rates for different pillars sizes, which was shown to affect the final mechanical behaviour.

We present a methodology to prepare acrylate micropillars via UV-curing on which micro-compression is performed by using a nano-indenter. In order to understand whether the layer thickness affects the properties of the printed product, cylindrical micropillars with length to diameter ratio $1: 1$, and diameters ranging from 40 to $80 \mu \mathrm{m}$, are fabricated and tested under uniaxial compression. Although the micropillars diameter range is small, a glassy polymer can show size-dependent mechanical behaviour at these length scales due to the intrinsic material heterogeneity. In fact, Wang and Guruprased et al. $[10,9]$, have indicated that glassy polymers exhibit intrinsic size effects on the yield strength during microcompression experiments. Wang et al. [9] have found a significant increase in yield and fracture strength with decrease in pillar diameter. This effect has been attributed to the lack of weak bonds in smaller samples. Guruprasad et al. [10] also reported that smaller pillars show a higher yield stress, that could be attributed to the higher rate of cooling and non-uniform temperature distribution that occurs during the preparation of smaller samples.

In the present work, a mixture of $3 \mathrm{wt} \%$ photo-initiator in bisphenol-diacrylate monomer is used to fabricate specimens via stereolithography. To study the influence of the exposure time and post-curing treatments, one-layer thick miniature samples are required to avoid an inhomogeneous structure along the sample thickness. Therefore, we have developed an experimental methodology that allows to study the intrinsic mechanical properties, both in the elastic and plastic deformation regime, by using micropillars and dog-bone shaped samples. Furthermore, the analysis of the effect of the different processing conditions on the material properties provides a better understanding of the stereolithography process and it can be used for process optimization.

\section{Materials and methods}

\subsection{Materials}

The monomer used for this study is a bisphenol-A-ethoxylate diacrylate (EO/phenol 2) (Sigma-Aldrich, number average molecular weight $\mathrm{M}_{n}=512 \mathrm{~g} / \mathrm{mol}$ ). An initiator, 2,2-dimethoxy-2-phenylacetophenone (Irgacure 651, from Ciba Specialty Chemicals, $M_{n}=256 \mathrm{~g} / \mathrm{mol}$ ), is used to generate the free radicals needed to initiate the photo-polymerization reaction of the acrylate monomer, see Fig. 1 . Next to the acrylate, a common epoxy based material, SU-8 3025 (MicroChem), is used to investigate the reliability of micro-compression tests. 


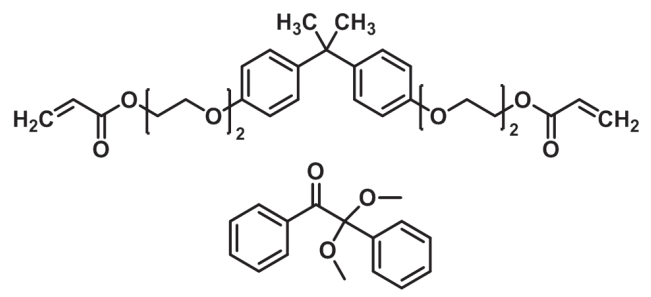

Figure 1: Chemical structure of EO/phenol 2 monomer and of Irgacure 651 photo-initiator.

\subsection{Preparation of pillars and tensile bars}

A UV-curable formulation is prepared by mixing $3 \mathrm{wt} \%$ of initiator in the acrylate monomer with an ultrasonic homogeniser. In this study, a consistent batch of the initial formulation has been prepared to avoid variations induced by the material composition. The steps involved in the preparation of pillars and tensile bars are shown in Fig. 2a. To improve the adhesion of the layer on the substrate, and to avoid defects in the final sample due to the presence of particles, 4-inch silicon wafers are initially cleaned with acetone and then dried with nitrogen gas. Spin coating is used to dispense the formulation on the substrate and to obtain a thin homogeneous layer. To prepare one single wafer, approximately $3 \mathrm{ml}$ of mixture is spun on the wafer; an initial spinning speed of $500 \mathrm{rpm}$ for $10 \mathrm{~s}$ is used to homogeneously disperse the liquid formulation over the entire surface. This is followed by a second step to obtain the desired layer with predefined thickness. It was verified that the spin coating process can be described as the coating of a Newtonian liquid on a rotating disk [11]:

$$
h=\sqrt{\frac{3 \eta_{0}}{4 \rho \omega^{2} t}},
$$

where $h$ is the film thickness, $t$ the spinning time, $\rho$ the liquid density, $\omega$ the spinning speed, and $\eta_{0}$ the solution viscosity. Using this model, the final thickness at each spinning speed can be predicted. The results are reported in the Supplementary information, Fig. S1.

The layer of the reactive UV-formulation is then locally UV-cured. Micropillars and tensile bars (ASTM D638 type1) are prepared by using a photomask. This mask is a quartz plate with transparent parts that allows UV-light to illuminate the sample with a well-defined pattern. In order to have a direct comparison between micro-compression and tensile tests, micropillars and dog-bone shaped samples are produced on one single wafer. A box has been fabricated to create an inert atmosphere that prevents oxygen inhibition, see Fig. $2 \mathrm{~b}$. The box is hermetically closed and the top part is made of quartz to prevent the reduction of the UV-light intensity. Once the wafer is placed in the box, the photomask is fixed in a frame and then aligned on top of the thin liquid layer. The presence of oxygen in the photo-curable polymer inhibits the propagation and termination reaction of the polymerization process. Therefore nitrogen is flushed for $20 \mathrm{~min}$ to create the inert atmosphere. To better replace the air present in the gap between wafer and photomask, magnets are used to lift up the photomask from outside while flushing nitrogen. The box is then placed in the UV-curing chamber and the material is exposed to the radiation of the LED light (wavelength 365 nm, Honle Group, LED Cube 100 model, Germany). The 


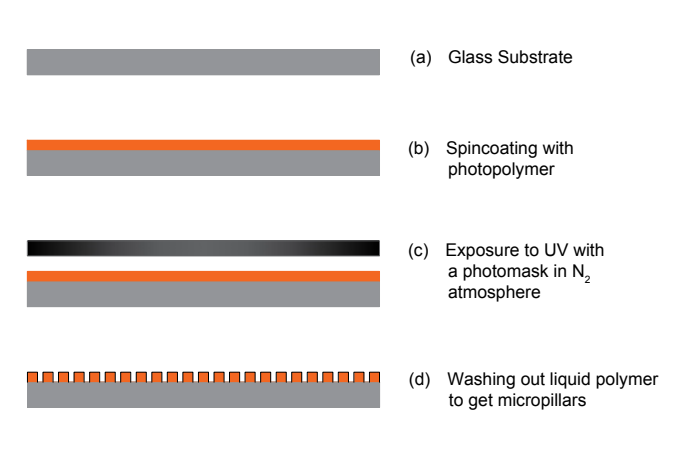

(a)

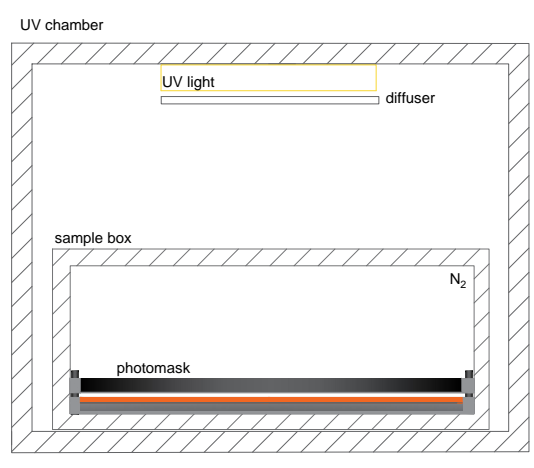

(b)

Figure 2: (a) Steps followed for the preparation of micropillars and tensile bars via stereolithograpy and (b) schematic representation of UV chamber.

light intensity at the sample position is uniform and equal to $7 \mathrm{~mW} / \mathrm{cm}^{2}$, as measured with a UV-meter.

Significant work has been done to find the optimal process conditions to obtain micropillars. Exposure time governs the shape of the cured micro-structured samples. In general, a short exposure time leads to formation of structures smaller than the mask openings due to insufficient exposure. On the other hand, long irradiation creates structures larger than the mask due to undesired exposure by scattering and reflection of the light in the regions which should not be photo-polymerized [12]. An exposure time of $1.5 \mathrm{~s}$ is found to be suitable to obtain samples with the desired shape. Next, the wafer is removed from the box, washed with acetone for $90 \mathrm{~s}$ to remove the uncured resin and dried with nitrogen. UV post-curing treatments are carried out in nitrogen atmosphere, at $7 \mathrm{~mW} / \mathrm{cm}^{2}$ for different irradiation times, and then thermal post-curing is performed in the oven at $150^{\circ} \mathrm{C}$ for $30 \mathrm{~min}$. At this point, tensile bars are carefully peeled off to get free-standing specimens. The micropillars are ready to be characterized and tested directly from the wafer.

In order to visualize the final shape and the surface properties, images of UV-cured micropillars are taken on a Scanning Electron Microscope (Fei Quanta 600F, ESEM) for which a gold coating is required to improve the image quality. A confocal microscope (Sensofar, Optical Imaging Profiler, Pl $\mu$ 2300) is used to characterize the geometry of the micropillars. An objective lens of 50X is used to obtain a 3D-optical profile of each micropillar that is successively processed to obtain the average height and diameter of the pillar. In this study, all the samples are first UV-cured for $1.5 \mathrm{~s}$ and then post-curing treatments are performed to study the influence of UV and thermal post-curing on the final properties.

\subsection{Samples characterization}

In order to understand the network structure of the cured acrylates, the first step in this study is the characterization of the curing kinetics. The degree of conversion is studied to assess the photopolymerization process and to understand under which conditions maximally-cured samples are obtained. The conversion is measured by Fourier transform infra-red spectroscopy (Spectrum Two FTIR 
Spectrometer, PerkinElmer) in the range of $4000-400 \mathrm{~cm}^{-1}$. After the photo-polymerization, 4 IRscans are performed to measure the consumption of the monomer during the reaction. The typical peaks used for the analysis of acrylate systems are the $C=C$ twist at wavenumber $810 \mathrm{~cm}^{-1}$, and the $\mathrm{C}=\mathrm{C}$ stretch at $1636 \mathrm{~cm}^{-1}$. In this study the wavenumber $810 \mathrm{~cm}^{-1}$ is used because the absorption peak is sharp and distinct [13]. The reduction of unsaturated bonds $(C=C)$ indicates the quantity of chemical bonds modified during the polymerization. To quantify the amount of monomer consumed, a comparison with the uncured mixture is made. While the reduction of the peak at $810 \mathrm{~cm}^{-1}$ indicates the progress of the reaction, the absorption at $829 \mathrm{~cm}^{-1}(\mathrm{C}=\mathrm{O}$ peak) does not change, which makes it useful as an internal standard. Therefore, this peak has been taken as reference to calculate the conversion. The degree of conversion $\alpha(t)$ is obtained by:

$$
\alpha(t)=\frac{\left[\frac{A_{810}^{\prime \prime}}{A_{829}^{\prime \prime}}\right]_{0}-\left[\frac{A_{810}^{\prime \prime}}{A_{829}^{\prime \prime}}\right]_{t}}{\left[\frac{A_{810}^{\prime \prime}}{A_{829}^{\prime \prime}}\right]_{0}} \cdot 100,
$$

where $\left[\frac{A_{810}^{\prime \prime}}{A_{829}^{\prime \prime}}\right]_{0}$ and $\left[\frac{A_{810}^{\prime \prime}}{A_{829}^{\prime \prime}}\right]_{t}$ represent the ratio of the second derivative of the absorption spectra at 810 and $829 \mathrm{~cm}^{-1}$, before and after UV exposure for time $t$. Second derivative spectra are useful to separate the signal from two or more components with overlapping peaks and to remove baseline errors [14]. This method is more robust as compared to using peak heights.

Dynamic mechanical thermal analysis (DMTA) is performed on the tensile bars using a TA instruments Q800. DMTA analysis is useful for characterizing polymers, since information on $\mathrm{T}_{g}$ and mechanical properties is obtained from one single experiment. The samples are heated at constant rate and, while heating, deformed with the same amplitude at a frequency of $1 \mathrm{~Hz}$, in a temperature range from $-50^{\circ} \mathrm{C}$ to $150^{\circ} \mathrm{C}$ with a heating rate of $3^{\circ} \mathrm{C} \cdot \mathrm{min}^{-1}$, a strain of $0.1 \%$ and a preload of $0.01 \mathrm{~N}$, which is in the linear viscoelastic range. The storage modulus $\left(E^{\prime}\right)$ and loss factor $(\tan \delta)$ are recorded as a function of temperature. Assuming simple affine network motion, the crosslink density $(v)$ of the UV-curable resin is determined by modulus measurements in the rubbery plateau from [15]:

$$
v=\frac{E^{\prime}}{3 R T},
$$

where the tensile storage modulus ( $\left.E^{\prime}\right)$ is obtained in the rubber plateau, $T$ is the temperature in $K$ corresponding to the storage modulus value, and $R$ is the universal gas constant.

Sol-content measurements are performed by weighing a cured film sample $\left(w_{0}\right)$ and then soaking it in the solvent (acetone) for $1 \mathrm{~h}$. The samples are then dried in a vacuum oven at $40^{\circ} \mathrm{C}$ until their weight is constant $\left(w_{2}\right)$. The sol-content of the cured films is then calculated as:

$$
\text { Sol }-\operatorname{content}(\%)=\frac{w_{0}-w_{2}}{w_{0}} \cdot 100 .
$$

The sol-content thus represents the residual soluble fraction of the photo-polymerized samples.

Nuclear magnetic resonance (NMR) analysis is performed to understand the effect of the solvent on the crosslinked network. Solutions of acetone with the uncured formulation, the film cured for $1.5 \mathrm{~s}$, and 
post-cured for $150 \mathrm{~s}$, are stirred for $90 \mathrm{~s}$. The soluble residue is redissolved in deuterated chloroform and successively analysed with a Bruker Advance-III $400 \mathrm{MHz}$ NMR source equipped with a BBFO probe (Bruker, Germany). Chemical shifts are reported in parts per million (ppm) referenced to an internal standard of residual proteosolvent $[D]$ chloroform $\left(\delta=7.26 \mathrm{ppm}\right.$ for ${ }^{1} \mathrm{H}$ relative to tetramethylsilane as an internal standard).

The molecular weight of the free small-length chains in the UV-cured polymer network is determined by means of gel permeation chromatography (GPC) after extraction with a solvent. Therefore, the cured films are stirred overnight in tetrahydrofuran (THF) at a concentration of $1 \mathrm{mg} / \mathrm{ml}$. The filtered solution is subsequently introduced in a Waters column equipped with a photo-diode detector and a refractive index detector (Waters 2996 and 2414). A polystyrene calibration curve is used to convert elution time to average molecular weight.

\subsection{Mechanical testing}

Two loading geometries are used for the mechanical testing procedures. Tensile tests are performed to study the effect of UV and thermal post-curing on the mechanical properties of a single layer. Additionally, to study the intrinsic mechanical response of the material under homogeneous deformation, micro-compression tests are performed on maximally-cured micropillars.

\subsubsection{Tensile properties}

Tensile tests are carried out on dog-bone shaped tensile bars, approximately $80 \mu \mathrm{m}$ thick, using a micro-tensile stage (DDS, Kammrath \& Weiss $\mathrm{GmbH}$ ). A load cell of $20 \mathrm{~N}$ is used for all the tests and the load is recorded for elongation rates between $10^{-5}$ to $10^{-3} 1 / \mathrm{s}$.

\subsubsection{Micro-compression - Nanoindentation}

The intrinsic behaviour is characterized by performing uniaxial compression tests using a Nano-indenter XP (MTS Nano-Instruments, Oak Ridge, TN). The cylindrical micropillars deform homogeneously into the plastic regime. During a test, load and displacement are constantly recorded as a flat-ended cone tip $\left(D_{\text {flat }}=300 \mu \mathrm{m}\right)$ is pressed onto the sample's surface with a constant speed. The mechanical response of the micropillars with diameters of 40,60 , and $80 \mu \mathrm{m}$ has been characterized. All tests have been performed under displacement control mode at penetration speeds ranging from 1 to 100 $\mathrm{nm} / \mathrm{s}$, corresponding to strain rates from $10^{-5}$ to $10^{-3} 1 / \mathrm{s}$.

\section{Results and Discussion}

To study the intrinsic behaviour of a maximally-cured layer of acrylate resin, we first characterize the curing kinetics of the material. UV-cured specimens have been prepared and UV and thermal post-treatments have been performed to study the evolution of the monomer conversion and the corresponding mechanical properties. Once the conversion and the glass-transition temperature do not 
change with time, a maximally-cured material is obtained. On these samples, micro-compression tests are performed to study the intrinsic mechanical properties of the system.

\subsection{Micropillar generation}

To characterize the curing kinetics and the mechanical properties representative for one single layer of acrylate UV-curable resin, proper sample preparation is crucial. The final shape of the sample is strongly correlated to the exposure time and the oxygen present in the environment. Free radicals generated during UV-radiation have a very short life-time and are quenched extremely rapidly if in contact with oxygen [4]. Verification of this oxygen effect is done by preparing specimens under two different curing conditions: in an inert atmosphere and air. The sample prepared in air shows a thin layer of uncured liquid material on top which leads to a reduction of the light intensity; the photo-polymerization rate slows down and, as a consequence, micropillars with smaller dimensions are observed, see Fig. 3.

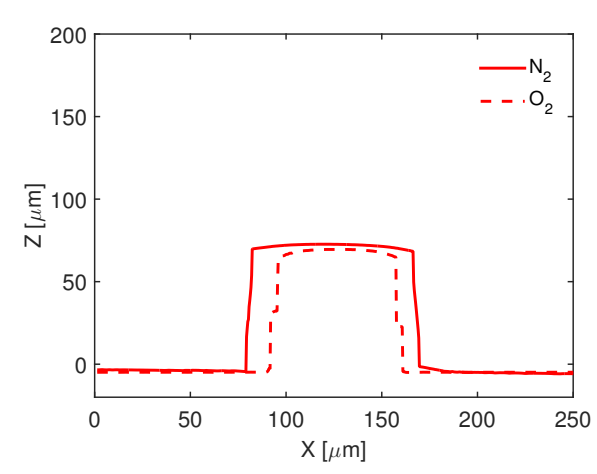

Figure 3: Confocal microscopy: effect of oxygen inhibition on the micropillars' geometry.

In order to obtain the desired final shape, all the specimens, micropillars and dog-bone shaped samples alike, are prepared by irradiating for $1.5 \mathrm{~s}$ at an intensity of $7 \mathrm{~mW} / \mathrm{cm}^{2}$. Next, the samples are cleaned with acetone to remove the uncured resin and successive post-curing treatments are performed. It should be noted that the pillar diameter is larger than the photomask openings due to the camera obscura effect. Although this effect influences the diameter, the divergent rays of light are not affecting the pillar shape. The SEM pictures reveal the desired geometry of the micropillars: a smooth surface and a perfect cylindrical shape are obtained. The contrast visible on the side surfaces of the pillar, see Fig. 4b, is due to the inhomogeneous gold distribution on the surfaces that occurs during the gold coating. 


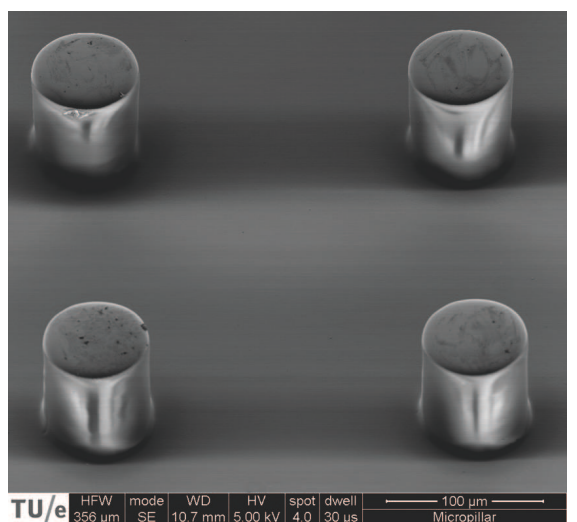

(a)

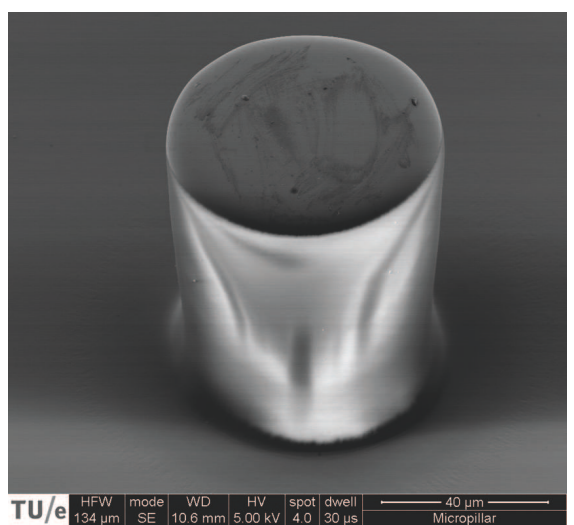

(b)

Figure 4: (a) SEM images of the array of micropillars and (b) a single micropillar of $80 \mu \mathrm{m}$.

\subsection{Effect of post-processing treatments}

\subsubsection{Monomer conversion evolution}

The photo-polymerization reaction leads to the formation of an insoluble 3D-network that determines the final mechanical properties of the material. Direct information about monomer consumption during the free-radical mechanism is obtained from Fourier transform infra-red spectroscopy (FTIR) by measuring the intensity of the signal associated with the vibrations of the acrylate group, see Fig. 5a. FTIR analysis on the initial liquid mixture, UV-cured and post-cured samples is carried out. In particular, samples first subjected to irradiation for $1.5 \mathrm{~s}$, are tested after UV post-curing of 50, 100, 150 and $200 \mathrm{~s}$ and thermal post-curing $\left(T_{P C}\right)$ for $30 \mathrm{~min}$ at $150^{\circ} \mathrm{C}$. The peak of the $\mathrm{C}=\mathrm{C}$ twist of the acrylate double bond is observed at $810 \mathrm{~cm}^{-1}$ and its evolution for UV and thermally post-cured samples is shown in Figs. $5 b$ and $5 c$. The peak intensity decreases significantly after the first UV-irradiation of $1.5 \mathrm{~s}$ and with UV post-curing it decreases further but with a much smaller amount. The FTIR spectra of samples previously UV post-cured and successively thermally post-cured are presented in Fig. $5 \mathrm{c}$. It can be concluded that a longer irradiation time leads to an increase of the double-bond consumption, whereas the thermal post-cured does not longer affect the curing kinetic, since an identical monomerto-polymer conversion is obtained.

The degree of conversion after UV post-curing, calculated as described in Section 2.3, no longer increases when the resin is irradiated for longer than $150 \mathrm{~s}$. Thermal post-curing on samples previously UV post-cured does not increase the monomer-to-polymer conversion, whereas it affects the conversion of the sample UV-cured for only $1.5 \mathrm{~s}$, where $\alpha(t)$ increases from 51 to $54.6 \%$. This is attributed to the increased mobility of the free radicals initially trapped within the vitreous network and their ability to further react with free monomers or polymerized chains, as presented by Bagis [16]. This effect results to be more pronounced in case of samples UV cured for only $1.5 \mathrm{~s}$, where a looser network is formed. Moreover, to confirm the reactivity of the system at higher temperature, the initial liquid formulation has been tested by a traditional DSC and the results reveal that at $150^{\circ} \mathrm{C}$ the thermally induced reaction occurs. The degree of conversion, shown in Fig. $5 d$, reaches a final value of $74 \%$ because of the vitrification that causes a limited mobility of the network [17]. 


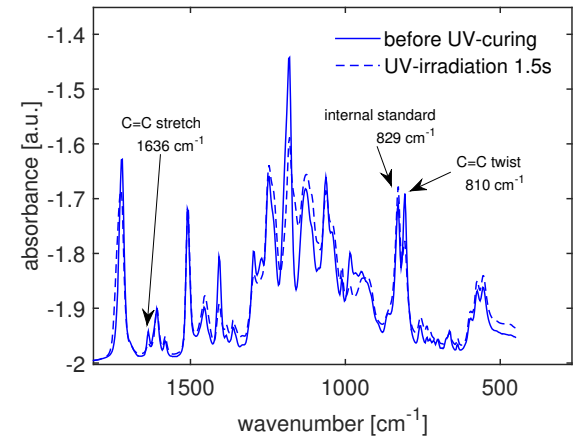

(a)

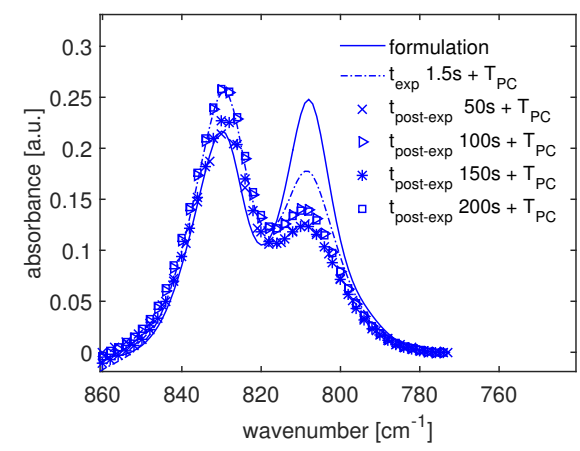

(c)

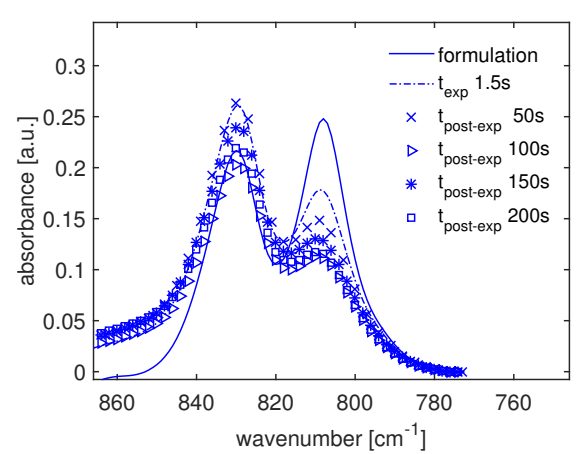

(b)

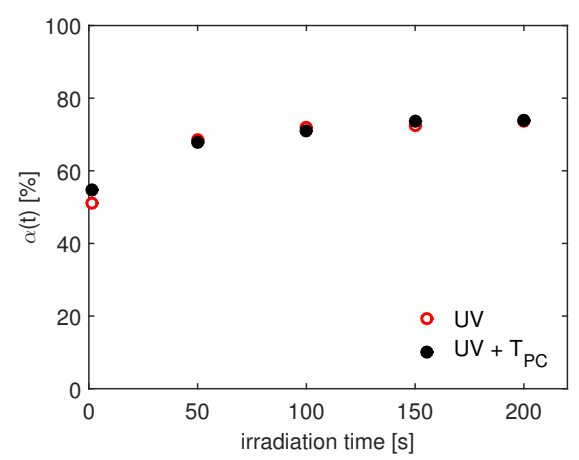

(d)

Figure 5: FTIR spectra: (a) full spectrum, (b) evolution with time of absorbance of $C=C$ twist of UV-post cured samples, (c) evolution with time of absorbance of $\mathrm{C}=\mathrm{C}$ twist of UV and thermally post-cured samples $\left(150^{\circ} \mathrm{C}\right.$ for $\left.30 \mathrm{~min}\right)$ and $(\mathrm{d})$ conversion versus time.

All the tests are performed on dog-bone samples of $80 \mu \mathrm{m}$ thick and repeated for three different locations along the length of the tensile bar. The provided data is the average of these three measurements and the standard deviation is approximately one percent. Moreover, tests on the top and bottom part of the samples have been conducted to precisely characterize the monomer conversion and check if a gradient is present throughout the layer thickness. No gradient is present within the $80 \mu \mathrm{m}$ thick layer (Supplementary Information, Fig. S2).

\subsubsection{DMTA results}

Dynamic mechanical analysis is carried out on samples which are UV and thermally cured. The storage and loss modulus, and the glass-transition temperature are determined. Generally, thermoset polymers have three distinct regions: the glassy region, characterized by a high storage modulus ( $E$ ') and low loss factor $(\tan \delta)$, a glass-transition region where $E^{\prime}$ decreases by a factor of $10-100$ and where the loss modulus ( $\left.\mathrm{E}^{\prime \prime}\right)$ and $\tan \delta$ reach a maximum, and a rubbery plateau region with a stable storage modulus. The temperature at which $\tan \delta$ reaches its maximum is defined here as the glass-transition temperature, $\mathrm{T}_{g}$. As an example, the temperature evolutions of $\mathrm{E}^{\prime}$ for a sample UV cured for $1.5 \mathrm{~s}$ and 
UV post-cured for $150 \mathrm{~s}$ with and without thermal post-curing are reported in Fig. 6a. All the cured samples exhibit the expected response, but the transition region is shifted to higher temperatures in case of post-treated samples. Previous work has shown that there is a correlation between the degree of

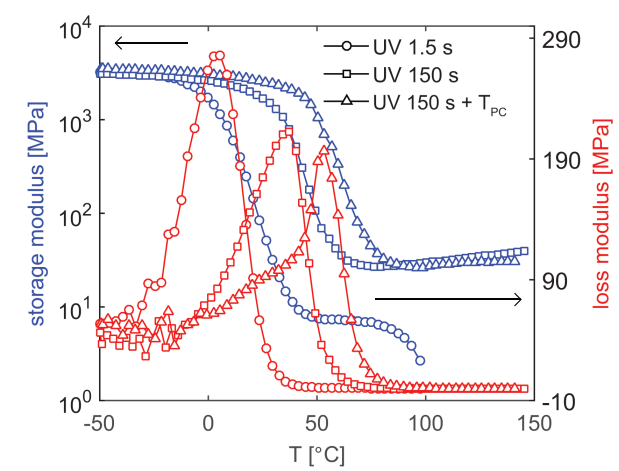

(a)

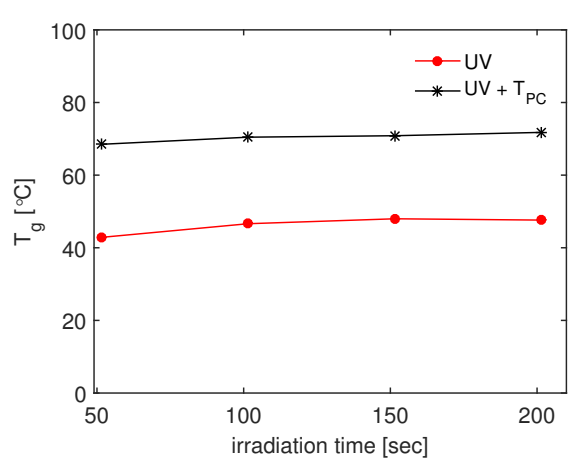

(b)

Figure 6: DMTA analysis: (a) the storage and loss modulus of the acrylate resin UV-cured for $1.5 \mathrm{~s}$ and UV and thermally post-cured, and (b) evolution of glass-transition temperature, $\mathrm{T}_{g}$, of UV and thermally $\left(150^{\circ} \mathrm{C}\right.$ for $\left.30 \mathrm{~min}\right)$ post-cured specimens.

curing and the glass-transition temperature $\left(T_{g}\right)$ [18]. The glass-transition temperature of the sample UV-cured for $1.5 \mathrm{~s}$ is found to be $25^{\circ} \mathrm{C}$, implying that a loose rubbery network is formed after the first UV-exposure. For UV and thermally post-cured specimens, a shift of the glass-transition regime is observed, therefore, samples with a higher $\mathrm{T}_{g}$ are formed. The evolution of $\mathrm{T}_{g}$ as a function of the postirradiation time of samples previously UV-cured for $1.5 \mathrm{~s}$ is presented in Fig. 6b. A slight increase in $\mathrm{T}_{g}$ is observed for longer post UV-irradiations and the results suggest that with $150 \mathrm{~s}$ maximally-cured material is obtained. Thermally post-cured samples show a further increase of $T_{g}$. This phenomenon can be linked to a rearrangement of the 3D-network while exposed to high temperature. The higher mobility during thermal post-curing leads to a rearrangement of the crosslinked network to a final more stable structure with higher $\mathrm{T}_{g}$.

The storage modulus in the rubbery plateau is used to calculate the concentration of network crosslinks. A higher value of $E^{\prime}$ indicates a higher crosslink density due to an increase of monomer conversion. This is reflected in the DMTA experiments performed, see Fig. 6a, where the rubber plateau for the sample only UV-cured for $1.5 \mathrm{~s}$ is lower than that of the UV and thermally post-cured samples. Here, the crosslink density increases from $0.88 \cdot 10^{-3}$ to $4.3 \cdot 10^{-3} \mathrm{~mol} / \mathrm{cm}^{3}$ respectively for $1.5 \mathrm{~s}$ UV-cured and maximally-cured samples, which is indicative of a highly crosslinked network [15]. Moreover, in accordance with the monomer conversion, see Fig. 5d, thermal post-curing no longer affects the rubbery plateau.

\subsubsection{Mechanical properties: tensile tests}

To investigate the effect of UV and thermal post-curing on the mechanical response, tensile tests are performed on the dog-bone shaped samples UV-cured for $1.5 \mathrm{~s}$, successively UV post-cured at different 
irradiation times and then thermally post-cured. The results for the UV post-cured specimens are shown

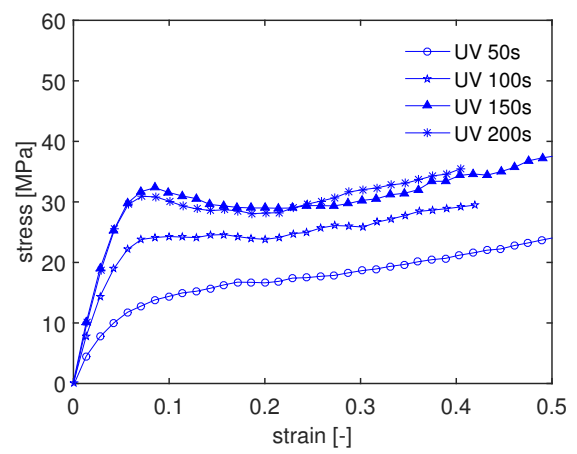

(a)

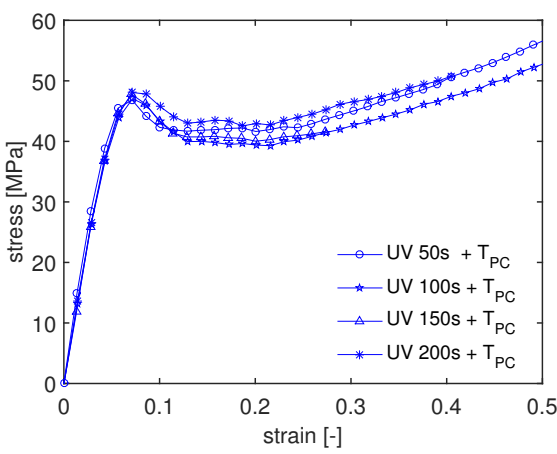

(b)

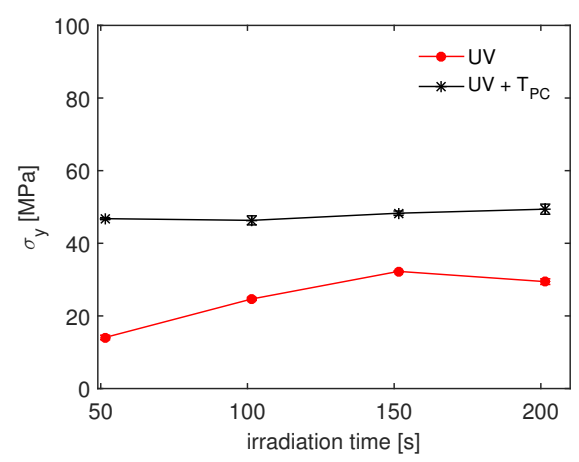

(c)

Figure 7: Engineering stress as a function of strain for (a) UV and (b) thermally post-cured samples measured at $23^{\circ} \mathrm{C}$. (c) Evolution with irradiation time of yield stress, $\sigma_{y}$, measured on UV and thermally $\left(150^{\circ} \mathrm{C}\right.$ for $\left.30 \mathrm{~min}\right)$ post-cured samples.

in Fig. 7a. Differences in mechanical response are mainly observed between samples post-irradiated for 50,100 and $150 \mathrm{~s}$. After $150 \mathrm{~s}$, the post UV-curing does not affect the mechanical response any further. This is in agreement with the conversion and glass-transition temperature results. By comparing Figs. $6 \mathrm{~b}$ and $7 \mathrm{c}$, it can be observed that the $\mathrm{T}_{g}$ follows a similar trend as the yield stress: $\mathrm{T}_{g}$ increases with increasing irradiation time. For this reason, in the glassy region the storage modulus already starts decreasing below $23^{\circ} \mathrm{C}$ for samples with lower irradiation time. As a result, a lower initial slope and a decrease of yield stress are observed, see Fig. 7a. Moreover, in agreement with Fig. 6b, after thermal post-curing, the stress versus strain results in Fig. $7 \mathrm{~b}$ are the same for all the samples. The thermal treatment leads to an increase of the glass-transition temperature, erases the differences caused by previous UV post-curing, and therefore, an increase in yield stress is observed, as shown in Fig. 7c. The difference results from an increase of chain interactions due to the possibility for radicals to react with a near neighbor during thermal post-curing, which can further limit the mobility of the network. Moreover, with thermal post-treatment, the network reaches a thermodynamically more stable structure and, consequently, an increase of both the glass-transition temperature and the mechanical response are observed. 


\subsection{Intrinsic mechanical response: micro-compression tests}

Micro-compression experiments are performed to characterize the intrinsic mechanical properties. Micropillars are prepared to determine the local mechanical behaviour of maximally-cured acrylate resin. All the specimens are prepared as explained in Section 2.2: the first irradiation of $1.5 \mathrm{~s}$ allows the formation of micropillars and, after the cleaning step with acetone, UV post-curing for $150 \mathrm{~s}$ is performed to obtain maximally-cured resin, as suggested by the $T_{g}$, conversion, and yield-stress evolutions. Further thermal post-curing is also carried out on these samples. Uniaxial compression tests are performed on maximally UV and thermally post-cured micropillars to investigate the material behaviour under homogeneous deformation by using a flat-ended tip of $300 \mu \mathrm{m}$. This geometry can cause an inexact alignment of the tip with respect to the sample's surface. In this study the misalignment is prevented by using a tilt stage, as reported in the work of Pelletier at al. [19].

At the start of the uniaxial compression test, the pillars are connected to the wafer substrate and this could induce undesired shear components in the deformation. However, SEM pictures of the micropillars obtained after compression show that the cylindrical shape is maintained, without the presence of buckling and distortions, see Fig. 8. This is caused by detachment of the pillars from the substrate due to limited adhesion strength. Therefore, the load-displacement data are directly converted to true stress-true strain curves.

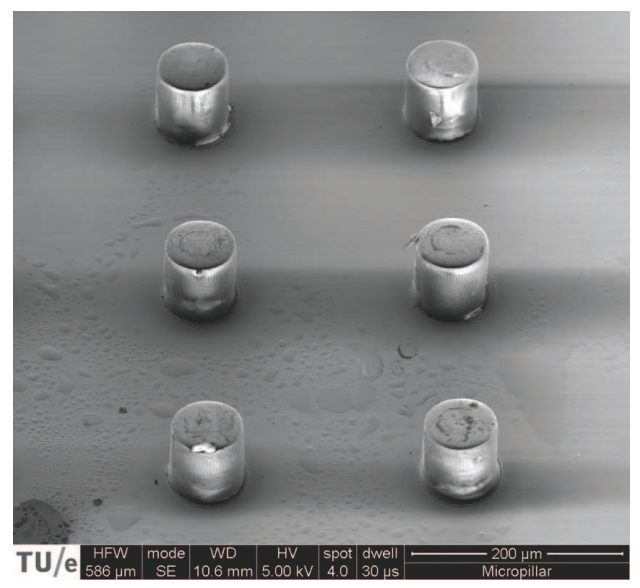

(a)

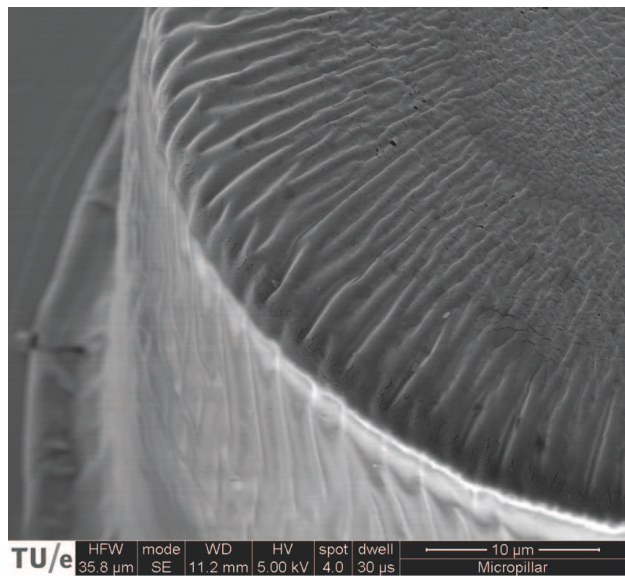

(b)

Figure 8: SEM images of (a) an array of compressed micropillars and (b) a detail of compressive failure of a maximally-cured pillar.

Initially, micro-compression experiments are performed on micropillars with a diameter of $80 \mu \mathrm{m}$ at different deformation rates to investigate the effect of strain rate. Fig. 9 shows true stress-true strain curves of micropillars with identical diameter subjected to several compression speeds. Five independent microcompression experiments have been performed for each compression speed and the curves are highly reproducible (see Fig. S3 in Supplementary Information). Fig. 9 indicates that the compressive yield stress increases with increasing compression speed. With increasing strain rate, a higher molecular mobility is required for yielding and this is achieved at a higher stress level. 


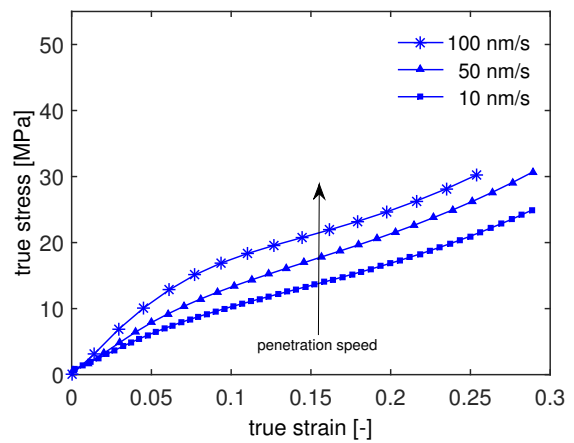

Figure 9: The true stress versus true strain response of micropillars for different penetration rates, measured at $23^{\circ} \mathrm{C}$.

The influence of strain rate and temperature on the yield stress can be described using Eyring's flow theory [20]:

$$
\sigma_{y}=\frac{k T}{V_{d}^{*}}\left(\ln \frac{2 \dot{\varepsilon}}{\dot{\varepsilon}_{0}}+\frac{\Delta U}{R T}\right),
$$

where $k$ is the Boltzmann constant, $R$ the universal gas constant, $T$ the temperature in $[K], \dot{\varepsilon}$ the applied strain rate, $V_{d}^{*}$ the activation volume, $\Delta U$ the activation energy and $\dot{\varepsilon}_{0}$ the rate constant. The dependency on strain rate is defined by $k T / V_{d}^{*}$. Introducing the zero-viscosity $\eta_{0}$ [21]:

$$
\eta_{0}=\frac{\sigma_{0}}{\dot{\varepsilon}_{0}} \exp \left(\frac{\Delta U}{R T}\right)
$$

and setting $\Delta U$ to zero because all the tests are performed at a single temperature, the yield kinetics is defined as:

$$
\sigma_{y}=\frac{k T}{V_{d}^{*}} \ln \left(\frac{2 \dot{\varepsilon} V_{d}^{*} \eta_{0}}{k T}\right),
$$

where

$$
\sigma_{0}=\frac{k T}{V_{d}^{*}} .
$$

The experimental results and the description by Eq. 7 are shown in Fig. 10a. The UV post-cured and thermally post-cured samples display an identical strain-rate dependence. The Eyring model describes the results for different post-curing conditions, with one value for $V_{d}^{*}$ and an increasing zero viscosity for increasing glass-transition temperature, see Table 1.

The same fit is made for tensile tests performed at different strain rates on samples cured under identical conditions. When the evolution of the yield stress is compared with that from the compression tests, a large unexpected discrepancy appears, see Fig. 10b. Both micropillars and tensile bars are prepared on one single silicon wafer to avoid variations in the material composition. This discrepancy can depend on the process conditions by which the samples are prepared: the wafer, after the first UV-irradiation, is cleaned with acetone to remove the uncured part. This solvent will affect the composition of the cured polymer and this effect can be more pronounced in case of samples with a larger surface-to-volume ratio. The explanation for the large difference observed between tensile and compression behaviour for 
Table 1: Parameter values

\begin{tabular}{l|cc} 
& $\mathbf{V}_{d}^{*}\left[\mathbf{n m}^{3}\right]$ & $\eta_{0}[\mathbf{P a} \cdot \mathbf{s}]$ \\
\hline Pillars UV & & \\
Pillars UVt-curing $\mathbf{U} \mathbf{V V}_{\text {post-curing }} \mathbf{1 5 0} \mathbf{~ s}$ & 2.2 & $6 \cdot 10^{11}$ \\
Tensile bars $\mathbf{U} \mathbf{T}_{\text {post-curing }} \mathbf{1 5 0} \mathbf{s}+\mathbf{T}_{P C}$ & 2.2 & $20 \cdot 10^{12}$ \\
& 2.2 & $75 \cdot 10^{18}$
\end{tabular}

the acrylate system, is sought in size differences of the samples. To study the size effect, compression tests are performed on micropillars with diameter and height of 40,60 , and $80 \mu \mathrm{m}$. Micropillars with smaller dimensions exhibit a drastic change in yield stress and post-yielding behaviour, see Fig. 11. It is evident that smaller micropillars show a reduction of the yield stress and a decrease in strain hardening. This can be attributed to a lower crosslink density of the network [22]. Therefore, the higher stressstrain response observed for tensile experiments, is hypothesized to be caused by differences in the network formed during the sample preparation.

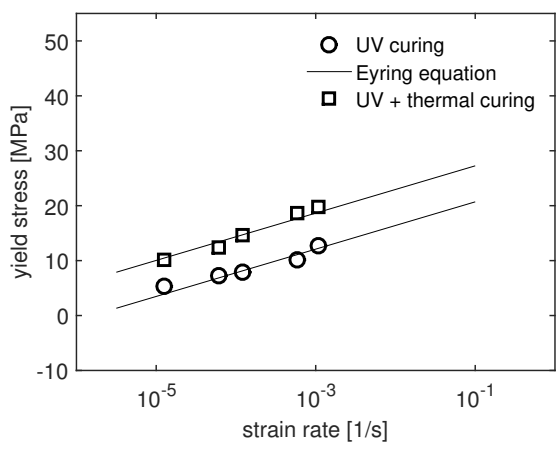

(a)

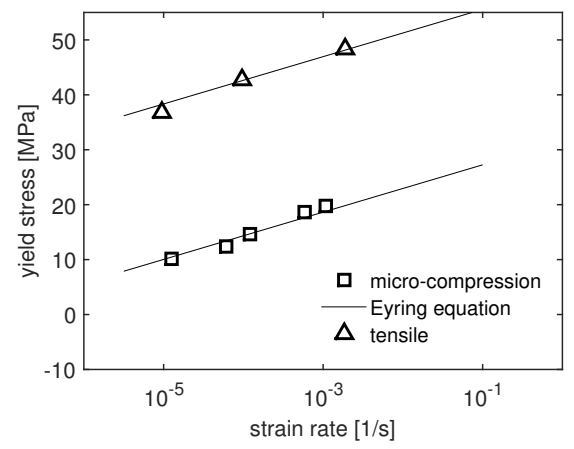

(b)

Figure 10: (a) The yield kinetics of UV and thermally post-cured micropillars and (b) the yield kinetics of micropillars and tensile bars prepared under the same curing conditions: UV for $150 \mathrm{~s}$ and thermal post-curing.

To further verify that the observed differences between tensile and compression results are indeed material related and not caused by the difference in characterization technique, the reliability of the nano-indentation technique is investigated by tests on a common epoxy based material. The microcompression experiments are validated with SU-8 3025 photoresist. The reported values for the modulus of SU-8 vary depending upon the process conditions, such as soft-baking time, exposure time, post baking and hard baking $[23,24]$. The yield kinetics and the evolution of the elastic modulus with strain rates of the tested SU-8 are shown in Fig. S4 in the Supplementary Information. The modulus values obtained in micro-compression are in accordance with the $2 \mathrm{GPa}$ given in the manufacturer datasheet of the SU8-3000 series and thus corroborate the reliability of the micro-compression technique. 


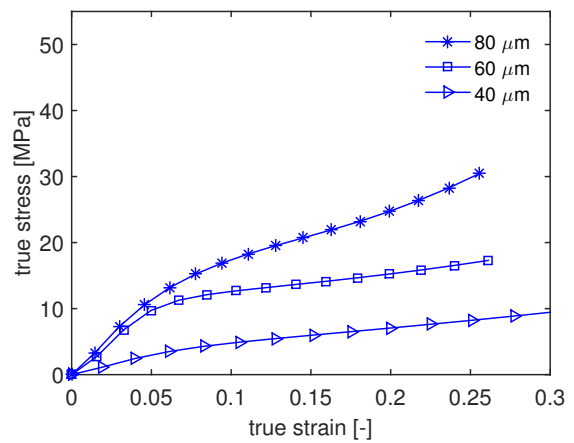

Figure 11: True stress-true strain response of micropillars of 80,60 , and $40 \mu \mathrm{m}$ at strain rate of $10^{-3}$ $\mathrm{s}^{-1}$ measured at $23^{\circ} \mathrm{C}$.

\subsection{Effect of acetone on the final morphology}

\subsubsection{Sol-content}

After a careful analysis of the sample preparation, it was hypothesized that the washing step, to remove excess monomer, influences the properties. Tests are performed to study the amount of soluble residue in the UV-cured network of samples prepared without using any solvent. Sol-content fraction is determined after the immersion of the cured samples in solvent (acetone) for one hour. After the initial UV-curing of $1.5 \mathrm{~s}$, the network contains a remaining $19 \%$ of soluble fraction, while with the next UV and thermal post-curing treatments, this reduces to respectively 9 and $2 \%$. The tests confirm that UV and thermal post-curing leads to additional reaction of uncured molecules. To obtain a better idea about the composition of the soluble part, the solvent plus the residue is analysed to determine whether the remaining components are monomers or photo-initiator molecules.

\subsubsection{NMR spectroscopy}

To investigate the composition of the washing solvent, specimens UV-cured for $1.5 \mathrm{~s}$ and UV postcured for $150 \mathrm{~s}$ are immersed in acetone for $90 \mathrm{~s}$ and the solutions are then filtered and examined with NMR-spectroscopy, see Fig. 12. The results were compared with that for the pure monomer. Peaks at 6.1-6.5 ppm prove the existence of acrylate double bonds in the solution, and they are more pronounced in the solution prepared from films UV-cured for $1.5 \mathrm{~s}$ rather then the ones UV post-cured for $150 \mathrm{~s}$. Moreover, peaks at 7.5-8 ppm are related to presence of photo-intitiator molecules in the solution. In particular, the concentration of the soluble material in the washing solvent results to be $21 \%$ for the $1.5 \mathrm{~s}$ UV-cured sample and $7 \%$ for the UV post-cured sample. These results suggest that by rinsing the sample with acetone, not only the uncured liquid resin around the cured features is washed away, but also monomers and photo-initiator are removed from the crosslinked network. It is known that the resin with a higher monomer conversion displays a higher glass-transition temperature and yield stress, see Fig. $6 \mathrm{~b}$ and $7 \mathrm{a}$. Therefore, the reduction in yield stress observed in smaller samples, can be related to the formation of a loose polymer network that might possibly be due to the higher amount of monomer rinsed from the polymer network per unit volume. 


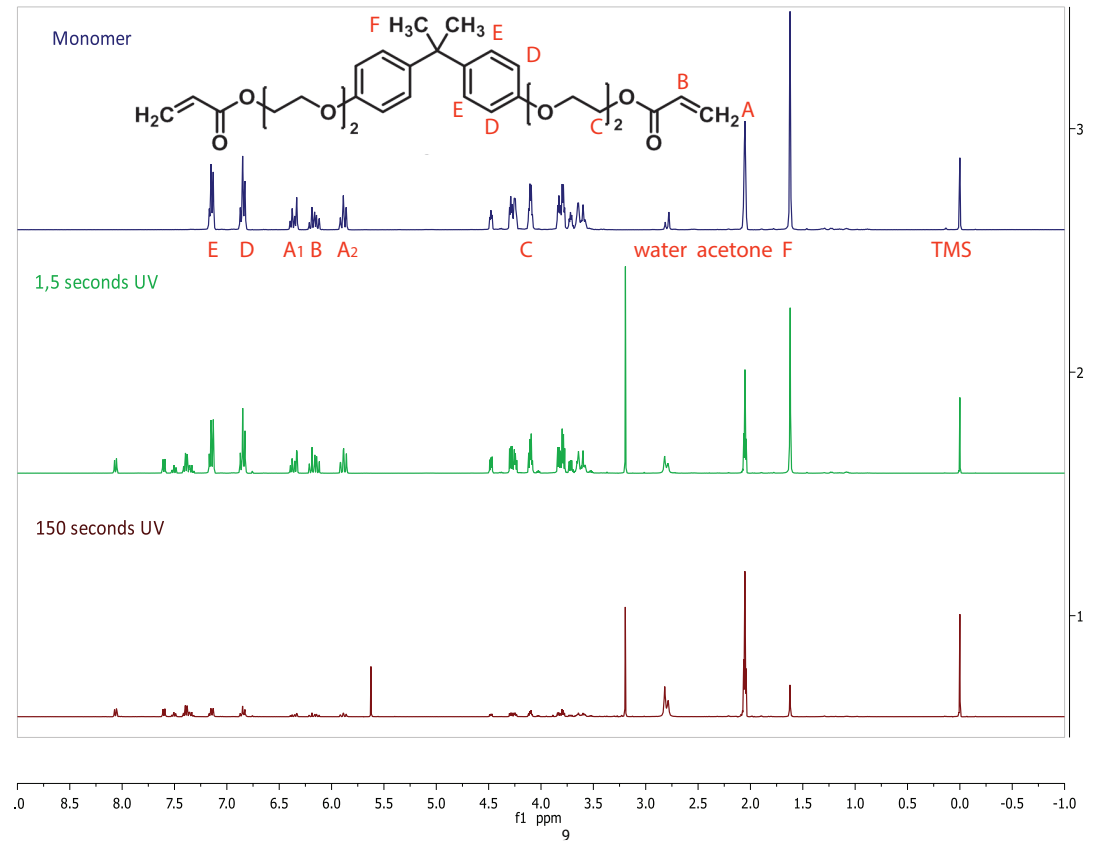

Figure 12: NMR spectra of solutions with monomer (top), washing solvent from film UV-cured $1.5 \mathrm{~s}$ (middle) and film UV post-cured for $150 \mathrm{~s}$ (bottom).

\subsubsection{Gel permeation chromatography}

The presence of small-length chains in the final crosslinked polymer can explain the role of posttreatments and the effect of the solvent on the 3D-network. In order to better understand how the solvent affects the polymer network, GPC analysis is performed on the soluble content in cured samples extracted with THF. Two UV and thermally post-cured films have been prepared: one with a washing step after the first $1.5 \mathrm{~s}$ UV-irradiation and the other one without using any solvent. Fig. 13a displays the GPC curves of the starting mixture, initiator, and monomer. The calculated average molecular weights are $\mathrm{M}_{w}=1014$ and $\mathrm{M}_{w}=120 \mathrm{~g} / \mathrm{mol}$, related to retention times of 18.5 and 21 min respectively. The two values are attributed to the presence of monomers, oligomers, and initiator in the formed network. When considering the thermally post-cured samples, no peak at $21 \mathrm{~min}$ is observed, therefore no more free radicals are present in the cured polymer, see Fig. 13c. Apparently, even though the monomer-to-polymer conversion values are identical for UV and thermally post-cured samples, thermal post-curing increases the network mobility and leads to a further reaction of radicals. The GPC results in Fig. 13b indicate that free monomer and oligomers are still present in the polymer network and the higher peak intensity of samples prepared without solvent, confirms the effect observed before. When the specimens are cleaned with acetone, molecules of monomer and photo-initiator are rinsed from 
the pre-formed network. As a result, GPC tests show a lower peak intensity for samples treated with solvent because less monomer is present in the final network.

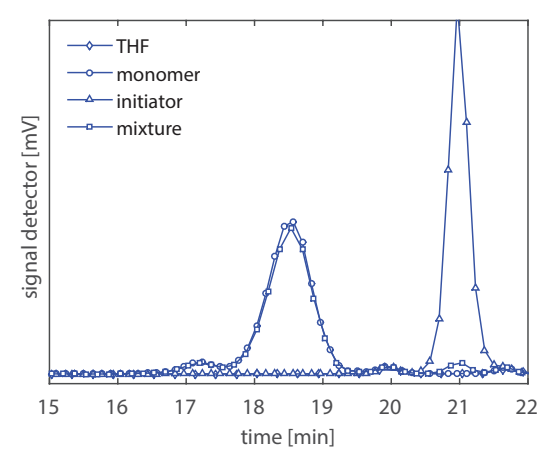

(a)

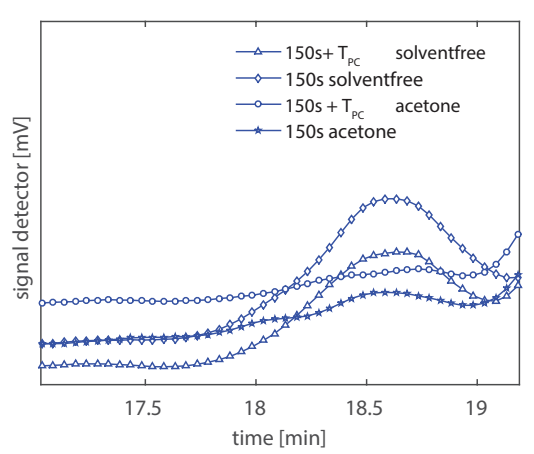

(b)

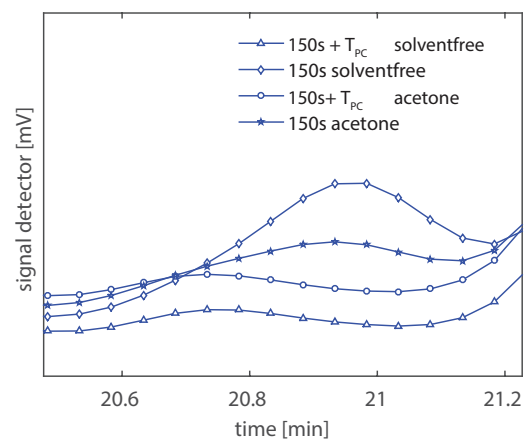

(c)

Figure 13: GPC curves for (a) initial liquid mixture, liquid monomer, photo-initiator and reference solvent (THF), (b) acrylate monomer and (c) photo-initiator spectra for THF extraction from UV and thermally post-cured films prepared without solvent, and film cleaned with acetone after $150 \mathrm{~s}$ of UV-exposure.

This effect of the solvent is more pronounced when smaller samples with a larger surface-to-volume ratio are washed with acetone. A larger amount of uncured monomer and photoinitiator is removed from the UV-cured network and, as a consequence, when the sample is post-cured, there are no more free monomers in the network. This results in a looser crosslinked network and, as a consequence, in lower values of the yield stresses. This phenomenon can also explain the decrease in strain hardening that appears when micro-compression tests are performed on smaller micropillars. In case of samples with a smaller surface-to-volume ratio, the space in the UV-cured network is filled with uncured monomers. As soon as the sample is UV post-cured, the UV-radiation activates the photo-initiators and the monomers start to polymerize. A graphical representation of the formation of the UV-cured network during the entire process is shown in Fig. 14. 


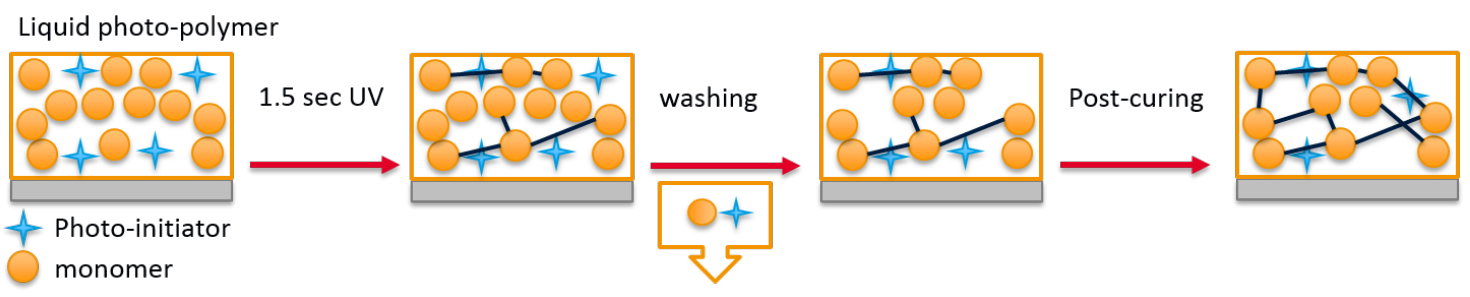

Figure 14: Schematic representation of the formation of the UV-cured network.

\section{Conclusions}

In this study, thermal and mechanical characterization of a single layer of UV-cured acrylate resin is performed. The results provide insight in the effect of process conditions, i.e. irradiation time and post-curing treatments, and the effect of solvent on the crosslinked structure. Bisphenol-A ethoxylate diacrylate monomer is used for the preparation of micron-sized samples via stereolithography. An inert environment and good control of exposure time are required for the preparation of micropillars and dog-bone shaped tensile bars. Monomer conversion, glass-transition temperature and yield-stress evolutions are evaluated as function of UV irradiation time. Post-curing treatments are needed to complete the photopolymerization process and to achieve ultimate mechanical properties.

Micro-compression tests are performed to characterize the mechanical response under uniaxial compression of maximally-cured micropillars. Cylindrical samples with diameters of 40,60 , and $80 \mu \mathrm{m}$ with aspect ratio $1: 1$ are tested. The measured yield stress depends linearly on the logarithm of strain rate, which is typical for polymer glasses. We also analysed the possible size effect on the yield stress. The mechanical response illustrates that for smaller micropillars the yield stress decreases. Moreover, tensile tests are performed on dog-bone shaped samples and the results show that, even if the samples are prepared under identical curing conditions, a higher yield stress is observed when compared with compression results. This discrepancy originates from the process conditions, i.e. the process by which the micropillars have been prepared, required the use of acetone to remove the uncured resin.

To study the effect of the solvent on the UV-cured network, sol-content analysis is performed on samples UV cured for $1.5 \mathrm{~s}$ and on those UV and thermally treated. The test confirms that $19 \%$ soluble fraction can still be removed from the network UV cured for $1.5 \mathrm{~s}$. Furthermore, to determine the composition of the soluble part, NMR analysis is conducted on the washing solvent. The spectra prove the presence of monomer and photo-initiator in the solution when the sample is treated with acetone. Therefore the washing protocol leads to a removal of uncured parts from the crosslinked network and this effect is more pronounced if smaller samples with a higher surface-to-volume ratio are treated. As a result, compared with solvent-free samples, a more loose network is formed during post-curing treatments due to the absence of uncured monomer. GPC analysis is conducted on maximally-cured material and the results prove the presence of monomers, oligomers, and photo-initiators in the crosslinked network. The curves of samples treated with solvent show a lower amount of free molecules. Thermal post-curing, compared with only UV post-curing, increases the network conversion, as observed in the reduction of the photo-initiator peak.

The considerations discussed in this work can be used to understand the roles of processing parameters involved in the stereolithography process, and mainly to probe the final mechanical properties represen- 
tative of one single layer of UV-cured acrylate resin by using micro-compression in combination with tensile experiments.

\section{Acknowledgement}

This work forms part of the research programme of the Brightlands Material Center (BMC). Professor R.P. Sijbesma and Dr. J.P.A. Heuts of the Macromolecular and Organic chemistry Department are kindly acknowledged for helpful discussions. The authors thank the groups Macromolecular and Organic Chemistry and Physical Chemistry for the use of the FTIR and GPC equipments. We acknowledge the students, Chiara Pastore and Mallika Rana, for helpful experimental analysis. 


\section{References}

[1] X. Yan and P. Gu. A review of rapid prototyping technologies and systems. Comput.-Aided Des., 28(4):307 - 318, 1996.

[2] P. J. Bartolo. Stereolithography: Materials, Processes and Applications. Sringer, 2011.

[3] L. Lu, J. Y. H. Fuh, A.Y.C. Nee, E. T. Kang, T. Miyazawa, and C. M. Cheah. Origin of shrinkage, distortion and fracture of photopolymerized material. Mater. Res. Bull., 30(12):1561 - 1569, 1995.

[4] C. Decker, T. N. T. Viet, D. Decker, and E. Weber-Koehl. Uv-radiation curing of acrylate/epoxide systems. Polymer, 42(13):5531 - 5541, 2001.

[5] M. J. Kanters, K. Remerie, and L. E. Govaert. A new protocol for accelerated screening of longterm plasticity-controlled failure of polyethylene pipe grades. Polymer Engineering \& Science, 56(6):676-688, 62016.

[6] H. E. Meijer and L. E. Govaert. Mechanical performance of polymer systems: The relation between structure and properties. Prog. Polym. Sci., 30(8):915 - 938, 2005. Plenary Lectures.

[7] A. Vitale, M. G. Hennessy, O. K. Matar, and J. T. Cabral. Interfacial profile and propagation of frontal photopolymerization waves. Macromolecules, 48(1):198-205, 2015.

[8] M. D. Uchic, D. M. Dimiduk, J. N. Florando, and W. D. Nix. Sample dimensions influence strength and crystal plasticity. Science, 305(5686):986-989, 2004.

[9] S. Wang, Y. Yang, L. M. Zhou, and Y.-W. Mai. Size effect in microcompression of epoxy micropillars. J. Mater. Sci., 47(16):6047-6055, 2012.

[10] T. S. Guruprasad, S. Bhattacharya, and S. Basu. Size effect in microcompression of polystyrene micropillars. Polymer, 98:118-128, 2016. Special Issue: Polymer Brushes.

[11] B. D. Hall, P. Underhill, and J. M. Torkelson. Spin coating of thin and ultrathin polymer films. Polymer Engineering \& Science, 38(12):2039-2045, 1998.

[12] V. C. Pinto, P. J. Sousa, V. F. Cardoso, and G. Minas. Optimized su-8 processing for low-cost microstructures fabrication without cleanroom facilities. Micromachines, 5(3):738-755, 2014.

[13] C. Decker and K. Moussa. A new method for monitoring ultra-fast photopolymerizations by realtime infra-red (rtir) spectroscopy. Die Makromolekulare Chemie, 189(10):2381-2394, 101988.

[14] L. Rieppo, S. Saarakkala, T. Närhi, H.J. Helminen, J.S. Jurvelin, and J. Rieppo. Application of second derivative spectroscopy for increasing molecular specificity of fourier transform infrared spectroscopic imaging of articular cartilage. Osteoarthritis Cartilage, 20(5):451 - 459, 2012.

[15] L. W. Hill. Calculation of crosslink density in short chain networks. Prog Org Coat, 31(3):235 243, 1997.

[16] Y. H. Bağis and F. A. Rueggeberg. Effect of post-cure temperature and heat duration on monomer conversion of photo-activated dental resin composite. Dent. Mater., 13(4):228 - 232, 1997. 
[17] C. Decker and C. Decker. Kinetic Study and New Applications of UV Radiation Curing. Macromol. Rapid Commun., 23:1067-1093, 2002.

[18] B. Herzog, D. J. Gardner, R. Lopez-Anido, and B. Goodell. Glass-transition temperature based on dynamic mechanical thermal analysis techniques as an indicator of the adhesive performance of vinyl ester resin. J. Appl. Polym. Sci., 97(6):2221-2229, 92005.

[19] C. G. N. Pelletier, E. C. A. Dekkers, L. E. Govaert, J. M. J. den Toonder, and H. E. H. Meijer. The influence of indenter-surface misalignment on the results of instrumented indentation tests. Polym. Test., 26(7):949 - 959, 2007.

[20] H. Eyring. Viscosity, plasticity, and diffusion as examples of absolute reaction rates. The Journal of Chemical Physics, 4(4):283-291, 1936.

[21] E. T. J. Klompen and L. E. Govaert. Nonlinear viscoelastic behaviour of thermorheologically complex materials. Mechanics of Time-Dependent Materials, 3(1):49-69, Mar 1999.

[22] H. G. H. van Melick, L. E. Govaert, and H. E. H. Meijer. On the origin of strain hardening in glassy polymers. Polymer, 44(8):2493 - 2502, 2003.

[23] S. Chung and S. Park. Effects of temperature on mechanical properties of su-8 photoresist material. J. Mech. Sci. Technol., 27(9):2701-2707, Sep 2013.

[24] R. Feng and R. J. Farris. Influence of processing conditions on the thermal and mechanical properties of su8 negative photoresist coatings. J. Micromech. Microeng., 13(1):80, 2003. 


\section{List of Tables}

1 Parameter values . . . . . . . . . . . . . . . . . . . . 16

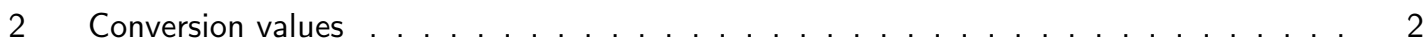

\section{List of Figures}

1 Chemical structure of EO/phenol 2 monomer and of Irgacure 651 photo-initiator. . . .

2 (a) Steps followed for the preparation of micropillars and tensile bars via stereolithograpy and (b) schematic representation of UV chamber. . . . . . . . . . . . . . . . . . 6

3 Confocal microscopy: effect of oxygen inhibition on the micropillars' geometry. . . . . 9

4 (a) SEM images of the array of micropillars and (b) a single micropillar of $80 \mu \mathrm{m}$. . . 10

5 FTIR spectra: (a) full spectrum, (b) evolution with time of absorbance of $\mathrm{C}=\mathrm{C}$ twist of UV-post cured samples, (c) evolution with time of absorbance of $\mathrm{C}=\mathrm{C}$ twist of UV and thermally post-cured samples $\left(150^{\circ} \mathrm{C}\right.$ for $\left.30 \mathrm{~min}\right)$ and $(\mathrm{d})$ conversion versus time.

6 DMTA analysis: (a) the storage and loss modulus of the acrylate resin UV-cured for 1.5 $\mathrm{s}$ and UV and thermally post-cured, and (b) evolution of glass-transition temperature, $\mathrm{T}_{g}$, of UV and thermally $\left(150^{\circ} \mathrm{C}\right.$ for $\left.30 \mathrm{~min}\right)$ post-cured specimens. . . . . . . . . . .

7 Engineering stress as a function of strain for (a) UV and (b) thermally post-cured samples measured at $23^{\circ} \mathrm{C}$. (c) Evolution with irradiation time of yield stress, $\sigma_{y}$, measured on UV and thermally $\left(150^{\circ} \mathrm{C}\right.$ for $\left.30 \mathrm{~min}\right)$ post-cured samples. . . . . . . . . . . . . . .

8 SEM images of (a) an array of compressed micropillars and (b) a detail of compressive failure of a maximally-cured pillar.

The true stress versus true strain response of micropillars for different penetration rates, measured at $23^{\circ} \mathrm{C} \ldots \ldots \ldots \ldots \ldots$

10 (a) The yield kinetics of UV and thermally post-cured micropillars and (b) the yield kinetics of micropillars and tensile bars prepared under the same curing conditions: UV for $150 \mathrm{~s}$ and thermal post-curing.

11 True stress-true strain response of micropillars of 80,60 , and $40 \mu \mathrm{m}$ at strain rate of $10^{-3} \mathrm{~s}^{-1}$ measured at $23^{\circ} \mathrm{C}$.

12 NMR spectra of solutions with monomer (top), washing solvent from film UV-cured 1.5 $\mathrm{s}$ (middle) and film UV post-cured for $150 \mathrm{~s}$ (bottom).

13 GPC curves for (a) initial liquid mixture, liquid monomer, photo-initiator and reference solvent (THF), (b) acrylate monomer and (c) photo-initiator spectra for THF extraction from UV and thermally post-cured films prepared without solvent, and film cleaned with acetone after $150 \mathrm{~s}$ of UV-exposure.

14 Schematic representation of the formation of the UV-cured network. . . . . . . . . . . 20

S1 Acrylate layer thickness after spin coating: symbols represent measured data, whereas continuous line represents the model. . . . . . . . . . . . . . . . . . . 1

S2 FTIR spectra of top and bottom of a tensile bar sample UV-cured for $1.5 \mathrm{~s}$. . . . . .

S3 The measured load-displacement response at a penetration rate of $100 \mathrm{~nm} / \mathrm{s}$ of five UV post-cured $80 \mu \mathrm{m}$ micropillars, showing the highly reproducible results. 
S4 (a) The yield kinetics of SU-8 micropillars and (b) elastic moduli obtained from uniaxial compression at different strain rates. 


\section{Supplementary information}

The final thickness of the liquid formulation mainly depends on the spin speed and spinning duration. The acrylate layer thickness as function of the spinning speed is shown in Fig. S1. The spinning duration is kept constant and equal to $30 \mathrm{~s}$ and the spin speed has been varied from 500 to $1400 \mathrm{rpm}$. The solid line represents the model described in Section 2.2 by the Eq. 1 .

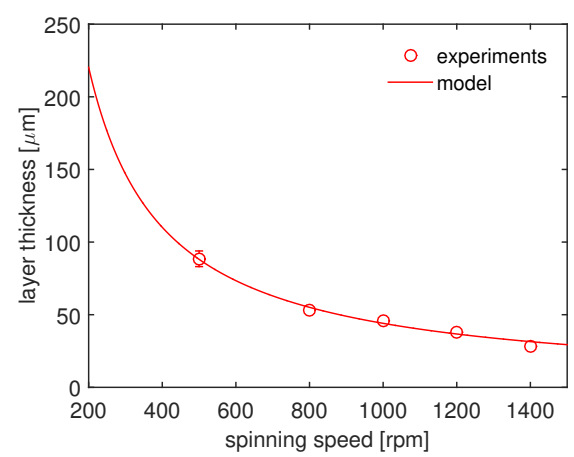

Figure S1: Acrylate layer thickness after spin coating: symbols represent measured data, whereas continuous line represents the model.

To check if a gradient in conversion is present throughout the cured layer thickness, FTIR analysis is performed on top and on the bottom of the layer. The results show that no gradient in conversion is present within the layer thickness, see Fig. S2.

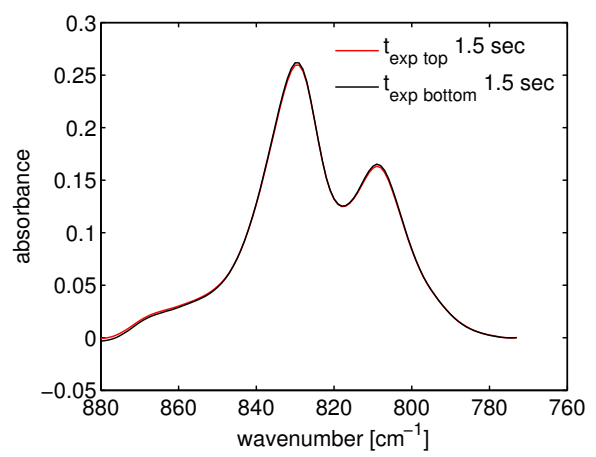

Figure S2: FTIR spectra of top and bottom of a tensile bar sample UV-cured for $1.5 \mathrm{~s}$.

The conversion after various irradiation times are reported in the Table 2.

Fig. S3 presents the load-displacement curves obtained by testing five different micropillars UV postcured for $150 \mathrm{~s}$ and shows the highly reproducible results.

To investigate the reliability of the nano-indentation technique, micro-compression tests are performed on micropillars of SU-8 3025 prepared via stereolithography. The samples are first exposed for $10 \mathrm{~s}$ to 
Table 2: Conversion values

\begin{tabular}{c|c} 
irradiation time [s] & conversion [\%] \\
\hline 1.5 & 51 \\
50 & 68 \\
100 & 72 \\
150 & 72.5 \\
200 & 74
\end{tabular}

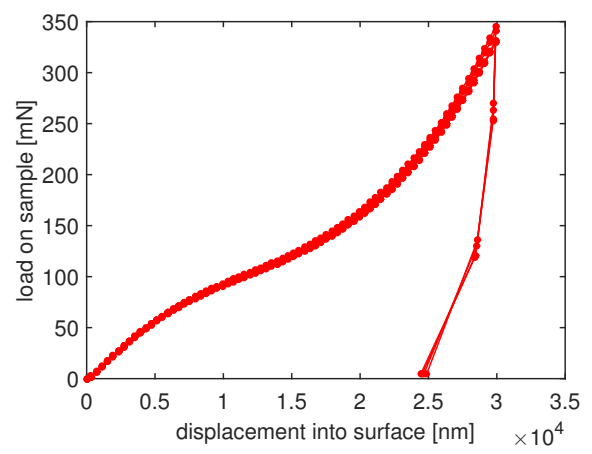

Figure S3: The measured load-displacement response at a penetration rate of $100 \mathrm{~nm} / \mathrm{s}$ of five UV post-cured $80 \mu \mathrm{m}$ micropillars, showing the highly reproducible results.

UV-light with an intensity of $15 \mathrm{~mW} / \mathrm{cm}^{2}$ and successively post-exposure baked for $1 \mathrm{~min}$ at $65^{\circ} \mathrm{C}$ and $3 \mathrm{~min}$ at $95^{\circ} \mathrm{C}$. Micropillars with a diameter of $20 \mu \mathrm{m}$ are tested under different strain rates and the evolutions of yield stresses and elastic modulus as function of the strain rates are reported in Fig. S4.

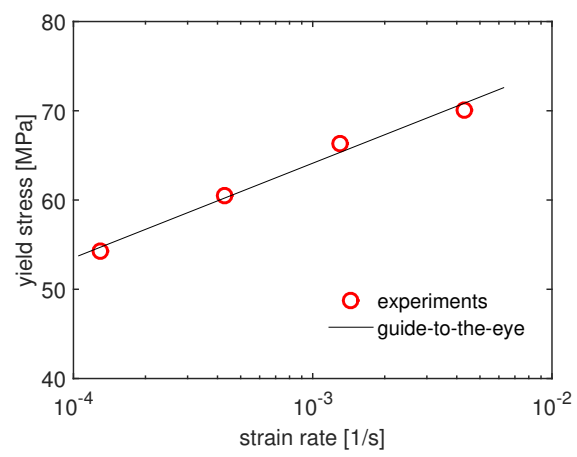

(a)

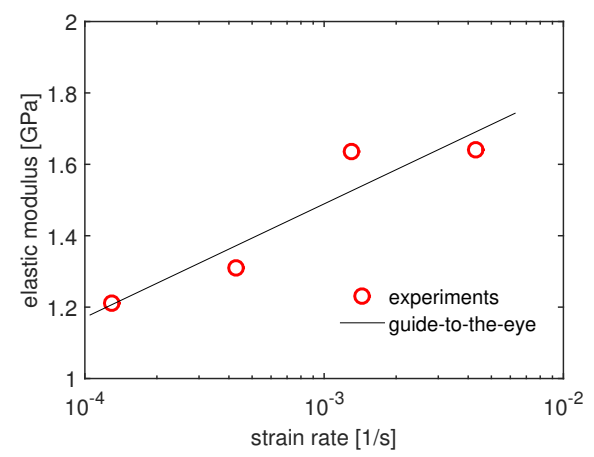

(b)

Figure S4: (a) The yield kinetics of SU-8 micropillars and (b) elastic moduli obtained from uniaxial compression at different strain rates. 